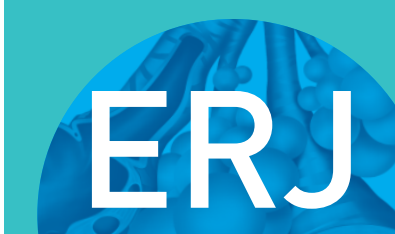

open research
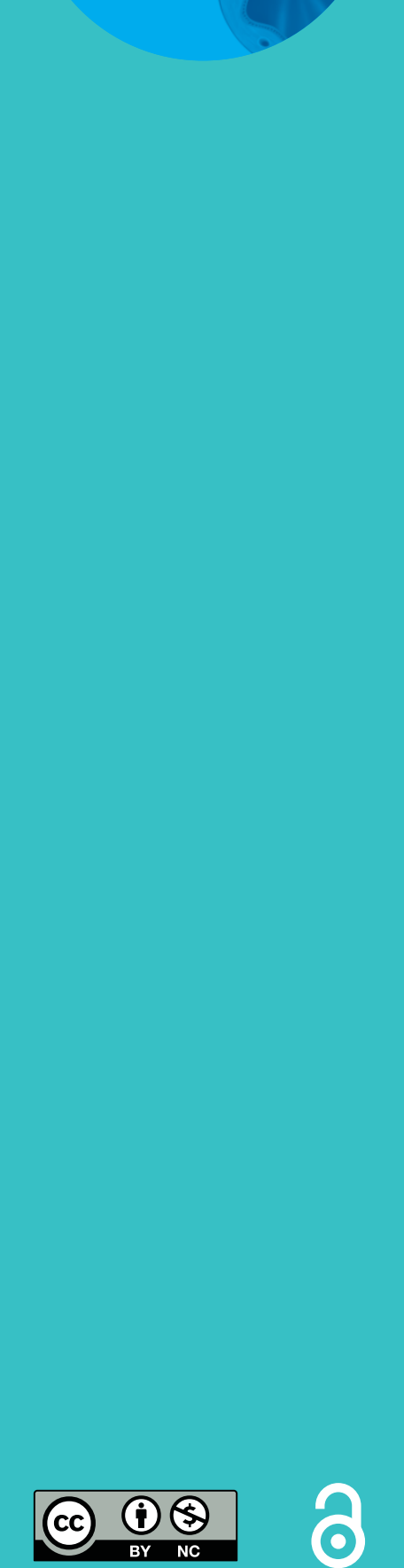

\title{
Standardised clinical data from patients with primary ciliary dyskinesia: FOLLOW-PCD
}

\author{
Myrofora Goutaki (10 ${ }^{1,2}$, Jean-François Papon ${ }^{3,4,5,6}$, Mieke Boon ${ }^{7}$, \\ Carmen Casaulta ${ }^{2}$, Ernst Eber $^{8}$, Estelle Escudier ${ }^{9}$, Florian S. Halbeisen (1) ${ }^{1}$, \\ Amanda Harris $^{10}$, Claire Hogg ${ }^{11}$, Isabelle Honore ${ }^{12}$, Andreas Jung (1) ${ }^{13}$, \\ Bulent Karadag ${ }^{14}$, Cordula Koerner-Rettberg ${ }^{15}$, Marie Legendre (1) \\ Bernard Maitre (10 ${ }^{16,17}$, Kim G. Nielsen ${ }^{18}$, Bruna Rubbo (10 ${ }^{10}$, Nisreen Rumman ${ }^{19}$, \\ Lynne Schofield ${ }^{20}$, Amelia Shoemark (1021, Guillaume Thouvenin (1022,23, \\ Hannah Willkins ${ }^{10}$, Jane S. Lucas (10 ${ }^{10}$ and Claudia E. Kuehni (i) ${ }^{1,2}$, for the \\ BEAT-PCD FOLLOW-PCD working group
}

ABSTRACT Clinical data on primary ciliary dyskinesia (PCD) are limited, heterogeneous and mostly derived from retrospective chart reviews, leading to missing data and unreliable symptoms and results of physical examinations. We need standardised prospective data collection to study phenotypes, severity and prognosis and improve standards of care.

A large, international and multidisciplinary group of PCD experts developed FOLLOW-PCD, a standardised clinical PCD form and patient questionnaire. We identified existing forms for clinical data collection via the Better Experimental Approaches to Treat PCD (BEAT-PCD) COST Action network and a literature review. We selected and revised the content items with the working group and patient representatives. We then revised several drafts in an adapted Delphi process, refining the content and structure.

FOLLOW-PCD has a modular structure, to allow flexible use based on local practice and research focus. It includes patient-completed versions for the modules on symptoms and lifestyle. The form allows a comprehensive standardised clinical assessment at baseline and for annual reviews and a short documentation for routine follow-up. It can either be completed using printable paper forms or using an online REDCap database.

Data collected in FOLLOW-PCD version 1.0 is available in real-time for national and international monitoring and research. The form will be adapted in the future after extensive piloting in different settings and we encourage the translation of the patient questionnaires to multiple languages. FOLLOWPCD will facilitate quality research based on prospective standardised data from routine care, which can be pooled between centres, to provide first-line and real-time evidence for clinical decision-making.

@ERSpublications

Standardised follow-up of PCD patients enables quality research with real-time data from routine care, providing evidence for clinical decision-making http://bit.ly/2PDfISF

Cite this article as: Goutaki M, Papon J-F, Boon M, et al. Standardised clinical data from patients with primary ciliary dyskinesia: FOLLOW-PCD. ERJ Open Res 2020; 6: 00237-2019 [https://doi.org/ 10.1183/23120541.00237-2019].

This article has supplementary material available from openres.ersjournals.com

Received: 6 Sept 2019 | Accepted after revision: 12 Dec 2019

Copyright $\odot$ ERS 2020. This article is open access and distributed under the terms of the Creative Commons Attribution Non-Commercial Licence 4.0. 


\section{Introduction}

Primary ciliary dyskinesia (PCD) is a rare, genetic, multi-organ disease characterised by dysfunction of motile cilia [1]. A European Respiratory Society taskforce performed a survey in 26 European countries in 2007-2009 and found that care of children with PCD was heterogeneous and decentralised [2, 3]. Although some countries have established designated PCD centres in the meantime, the situation has not changed substantially $[4,5]$. Management recommendations are based on expert opinion and observations from few patients, or are extrapolated from other chronic respiratory diseases such as cystic fibrosis (CF) and non-CF bronchiectasis [6,7]. We need better evidence on PCD clinical phenotypes, severity and prognosis and on the factors that influence these to improve the standards of care and tailor care to patient needs.

Availability of clinical data for PCD research is limited. There are few patient registries and these collect limited phenotypic data $[5,8]$. Conversely, detailed data are regularly collected in clinics during care of PCD patients, but not in a standardised way $[9,10]$. Retrospective data collection from charts leads to heterogeneous clinical data with many missing items; it is unreliable for symptoms and results of physical examination [11, 12]. Every clinic collects information in a different way, and even within clinics, recording of patient history and clinical examination varies between physicians and clinical specialties. In a systematic review of all publications describing clinical manifestations in PCD, we found that symptoms had been assessed in such a heterogeneous way that pooling of data or comparisons between studies was impossible [10]. This highlights the need for standardised collection of clinical data to be used in well-designed real-time clinical research.

To address these issues, we developed FOLLOW-PCD, a disease-specific form for standardised prospective data collection during routine clinical follow-up of PCD patients.

\section{Methods}

The protocol for the development of FOLLOW-PCD consisted of several steps (figure 1). This article describes in detail the first steps of the protocol and the resulting version 1.0 of FOLLOW-UP in English. In addition, it explains the next planned steps.

\section{Working group}

FOLLOW-PCD was developed by a multidisciplinary, international working group of the BEAT-PCD (Better Experimental Approaches to Treat PCD) COST Action network (www.beatpcd.org/) [13-15]. BEAT-PCD is a European-led network of $>250$ scientists and clinicians from 25 countries, coordinating research to improve the care and diagnosis of PCD. The FOLLOW-PCD working group consisted of 41 members of the BEAT-PCD network from 12 countries, mainly Europe. The composition of the group reflected the BEAT-PCD network: 24 paediatric respiratory physicians, three adult pulmonologists, one otolaryngologist, four diagnostic experts, two clinical nurse specialists, three physiotherapists and four epidemiologists (supplementary table S1). 26 clinical centres, with $>1000$ PCD patients under care in total,

Affiliations: ${ }^{1}$ Institute of Social and Preventive Medicine, University of Bern, Bern, Switzerland. ${ }^{2}$ Paediatric Respiratory Medicine, Children's University Hospital of Bern, University of Bern, Bern, Switzerland. ${ }^{3}$ AP-HP, Hôpital Kremlin-Bicetre, Service d'ORL et de Chirurgie Cervico-Faciale, Le Kremlin-Bicêtre, France. ${ }^{4}$ Faculté de Médecine, Université Paris-Saclay, Le Kremlin-Bicêtre, France. ${ }^{5}$ INSERM, U955, Créteil, France. ${ }^{6} \mathrm{CNRS}$ ERL 7240, Créteil, France. ${ }^{7}$ Dept of Paediatrics, University Hospital Gasthuisberg, Leuven, Belgium. ${ }^{8}$ Division of Paediatric Pulmonology and Allergology, Dept of Paediatrics and Adolescent Medicine, Medical University of Graz, Graz, Austria. ${ }^{9}$ Service de Génétique et Embryologie Médicales, Hôpital Armand-Trousseau, Assistance Publique-Hôpitaux de Paris, Paris, France. ${ }^{10}$ Primary Ciliary Dyskinesia Centre, NIHR Respiratory Biomedical Research Centre, University of Southampton, Southampton, UK. ${ }^{11}$ Primary Ciliary Dyskinesia Centre, Royal Brompton and Harefield Foundation Trust, London, UK. ${ }^{12}$ Assistance Publique-Hôpitaux de Paris, Hôpital Cochin, Service de Pneumologie, Paris, France. ${ }^{13}$ Division of Respiratory Medicine, University Children's Hospital of Zurich, Zurich, Switzerland. ${ }^{14}$ Dept of Pediatric Pulmonology, Marmara University, School of Medicine, Istanbul, Turkey. ${ }^{15}$ Dept of Paediatric Pneumology, University Children's Hospital of Ruhr University Bochum, Bochum, Germany. ${ }^{16}$ Centre Hospitalier Intercommunal de Créteil, Service de Pneumologie et de Pathologie Professionnelle, DHU A-TVB, Université Paris Est-Créteil, Créteil, France. ${ }^{17}$ INSERM U955, Institut Mondor de Recherche Biomédicale (IMRB) Equipe 04, Créteil, France. ${ }^{18}$ Danish PCD Centre Copenhagen, Paediatric Pulmonary Service, Copenhagen University Hospital, Copenhagen, Denmark.

${ }^{19}$ Al-Quds University, Faculty of Medicine, East Jerusalem, Palestine. ${ }^{20}$ Leeds Teaching Hospital NHS Foundation Trust Leeds, Leeds, UK. ${ }^{21}$ Tayside Respiratory Research Group, University of Dundee, Dundee, UK. ${ }^{22}$ Paediatric Pulmonary Dept, Trousseau Hospital APHP. Sorbonne Universities and Pierre et Marie Curie University, Paris, France. ${ }^{23}$ Sorbonne Université, INSERM, Centre de Recherche Saint-Antoine, CRSA, Paris, France.

Correspondence: Myrofora Goutaki, Institute of Social and Preventive Medicine, University of Bern, Mittelstrasse 43, 3012 Bern, Switzerland. E-mail: myrofora.goutakidispm.unibe.ch 


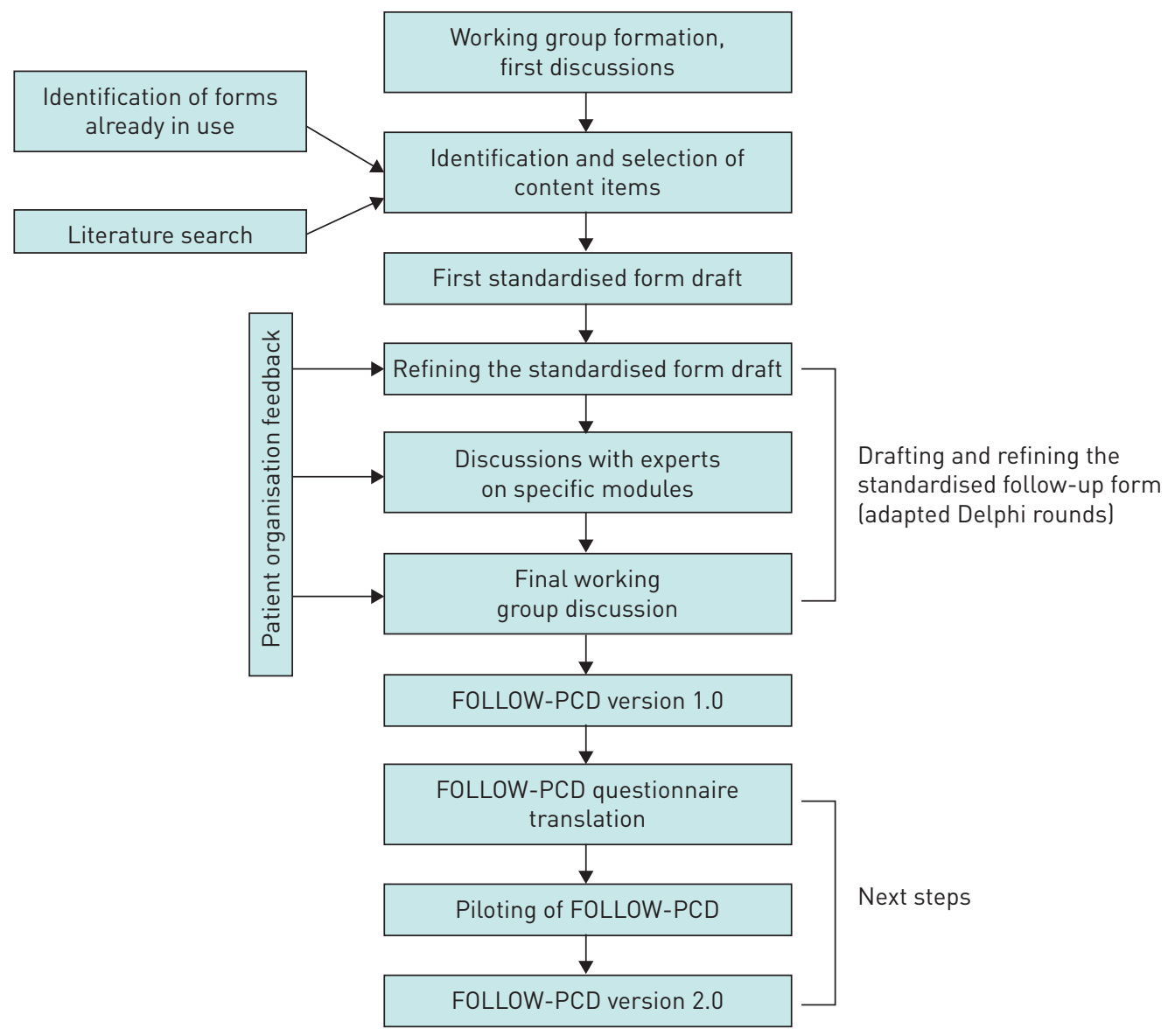

FIGURE 1 Development process of the standardised pulmonary ciliary dyskinesia (PCD) follow-up form (FOLLOW-PCD).

were represented in the working group by one or more team members. The project was developed and coordinated by the two co-principal investigators (MG and $\mathrm{CK}$ ) from the Institute of Social and Preventive Medicine (ISPM; Bern, Switzerland).

\section{Identification and selection of content items}

We contacted all members of the BEAT-PCD network to identify any forms that were already in use for data collection during clinical follow-up visits. We asked about PCD-specific forms and forms used for patients with other chronic respiratory diseases, e.g. CF and non-CF bronchiectasis. In addition, we performed a systematic literature search to find clinical studies of PCD patients that used a standardised clinical assessment form. We searched for studies published between January 1980 and April 2016 including published abstracts, without restrictions in language or study design.

Only eight centres, from five countries, out of the 22 countries participating in BEAT-PCD, used PCD-specific forms for the follow-up of patients. The literature search did not yield additional forms. We compared content and structure of the forms. These assessed roughly the same information, but not in a standardised way. The item categories contained in most forms were 1) summary of diagnostic information; 2) short clinical history; 3) summary of physical examination of the lungs; 4) lung function measurement; 5) results of microbiology testing; 6) results of imaging; and 7) recommended medication. Information on the upper airways, fertility and patient-reported outcomes were almost never included.

At the inaugural BEAT-PCD meeting in Southampton, UK (December 2015), participants exchanged ideas and opinions about content and format of the standardised follow-up form (figure 1) [13]. We agreed on the need for an extensive baseline form and a short form for regular follow-up. The advantages and disadvantages of different versions for paediatric and adult patients were discussed.

We then discussed the content items identified in face-to-face meetings and group telephone conferences with the working group and with patient representatives. This enabled the identification of additional 
items. Patient representatives provided specific feedback, particularly on the need to include patient-reported symptoms.

\section{Drafting and refining the standardised follow-up form}

Based on the final list of items, MG drafted the follow-up form. Several drafts were discussed in an adapted Delphi process to tailor its contents to different settings and make it appropriate for both clinical use and research (figure 1). $>80 \%$ of the working group members participated in every discussion round. Overall the process included four discussion rounds, where all members were invited to comment on the latest draft by 1) suggesting items to be removed or added and 2) commenting on the specific phrasing and wording of the included items, the sequence of the questions and the overall structure. The process involved discussions at BEAT-PCD conferences, telephone and skype calls and email communication. A main issue raised during discussions was structure. Related to the structure, the discussions focused on whether there should be two main versions, one for the baseline assessment and one for follow-up visits. As an alternative, we discussed a form with several distinct modules. This would permit completing all modules at baseline and during annual reviews and fewer modules during 3-monthly routine follow-up. The working group took into account the validated PCD quality of life questionnaires to avoid overlaps so that the instruments can be used together and complement each other [16-19]. After each round, MG resolved disagreements among participants, contacting them individually if required, and then made the next draft. Particular consideration was given to comments and suggestions by adult pulmonologists and experts on specialised topics. When there was no clear decision, different options were included in the revised draft asking the group to decide. Each decision was taken with agreement of the majority $(>80 \%)$ of the participants of each round. Details on specialised topics such as genetics, diagnostic testing, upper airways and physiotherapy were discussed with the experts before and during the group discussions. A final group discussion resulted in the development of version 1.0 of FOLLOW-PCD.

\section{Ethics and data agreements}

Local collaborators are responsible for obtaining ethics approval and informed consent in their country for use of FOLLOW-PCD to collect prospective clinical data and use it for research nationally and internationally.

\section{Results}

\section{Standardised PCD follow-up form (FOLLOW-PCD)}

We strived for an easy and practical instrument for clinical use, but also considered it important that it is comprehensive (box 1). The working group unanimously agreed to a modular structure, which permits different members of the multidisciplinary team (pulmonologists, otolaryngologists, diagnostic experts, physiotherapists, lung function technicians, etc.) to complete different modules (table 1). After agreeing on the modular structure, the working group decided to prepare two versions of the modules on patient-reported outcomes (symptoms and environment-lifestyle). Version A is formatted for completion by the physician during the clinical visit, similarly to the other modules. Version B collects the same information, but is formatted as a questionnaire, which can be completed directly by patients. In addition, we created age-adapted versions for these modules, because some symptoms and relevant exposures vary by age, for instance questions on active smoking for adults and adolescents versus parental smoking for children. We also decided to have FOLLOW-PCD available both as paper form and as an electronic database.

\section{Structure and content of FOLLOW-PCD}

Version 1.0 of FOLLOW-PCD consists of seven modules (table 1). It is possible to have a more extensive evaluation at baseline and annual reviews, when all modules are completed and a short documentation at routine (e.g. 3-monthly) follow-up, where only a few modules are used (box 1). Module 1 must be filled only at the first visit (at diagnosis or referral from other clinic) and includes demographic information, extensive diagnostic data, information on unchanging congenital abnormalities (e.g. situs inversus, cardiac defects) and a baseline medical history. The module can be updated at any time if patients have had additional diagnostic tests performed.

Modules 2-7 are designed for use at baseline and during routine follow-up visits. These modules include physical examination of the lungs, heart and upper airways, growth measurements, measurement of lung function, microbiology and imaging, information on hospitalisations, surgeries, prescribed treatments and medical history. Table 2 presents the content included in each module. Modules 6 and 7 contain patient-reported information on clinical symptoms and lifestyle (e.g. exercise and smoking).

For annual reviews, all modules are recommended. For routine 3-monthly follow-up visits, each clinic can use the modules that fit the local routine practice, using for example module 2 for the examination of 
BOX 1 FOLLOW-PCD: an instrument for standardised medical record keeping for patients with primary ciliary dyskinesia (PCD)

FOLLOW-PCD is:

- a standardised instrument for recording information collected during baseline and routine follow-up visits of patients with PCD

- developed by an interdisciplinary working group of adult and paediatric pulmonologists, otolaryngologists, physiotherapist and clinical nurse specialists

- a way of recording information on past and current medical history, physical examination, diagnostic tests and prescribed treatment

- a modular instrument, allowing to document extensive information at the time of diagnosis, and during annual reviews, and short updates during routine (e.g. 3-monthly) visits

- a flexible tool that can be completed by different healthcare professionals le.g. pulmonologists otolaryngologists, physiotherapists, nurses) and patients or caregivers themselves (data on symptoms, lifestyle and environment)

FOLLOW-PCD will allow the PCD community to:

- collect routine clinical data in a standardised prospective way

- cover most aspects that are relevant for management and care

- compare and pool data between centres for benchmarking and monitoring, compare diagnostic and prognostic investigations and treatments between centres and countries

- have a real-life representative dataset available for international monitoring and research

- perform high-quality real-time research on clinical questions such as phenotypes, severity and prognosis, or response to treatments in patients with PCD and to study the factors that influence them

- base clinical decision-making on real-life, contemporaneous and representative data based on large numbers of patients

lungs and heart, parts of modules 4 (growth and lung function, microbiology) and 5 (medication and physiotherapy) and module 6 administrated as a patient questionnaire or completed by the physician.

\section{Patient questionnaires}

For modules 6 and 7 there is the option to complete the physician forms (version A) or the patient questionnaires (version B), or both and compare them. There are three age-related versions of the questionnaires: one for adult patients, one for adolescents aged 14-17 years and one for the parents or caretakers of patients aged $<14$ years, with different questions on work or school attendance, physical activity and smoking.

\section{Electronic data entry}

In addition to the paper version, the standardised PCD follow-up form is available electronically through the Research Electronic Data Capture (REDCap) platform developed at Vanderbilt University (Nashville, TN, USA) [20]. Centres using the REDCap platform can enter prospective standardised clinical data that can be pooled for collaborative studies (box 2). REDCap is secure, widely used in academic research and allows data entry and extraction in various formats. We designed one central REDCap database, hosted and managed at ISPM Bern. All collaborators have personal access accounts, which allows access only to data from their centre. In the electronic version of FOLLOW-PCD, collaborators are invited to enter

TABLE 1 Modules included in the standardised pulmonary ciliary dyskinesia (PCD) follow-up form (FOLLOW-PCD)

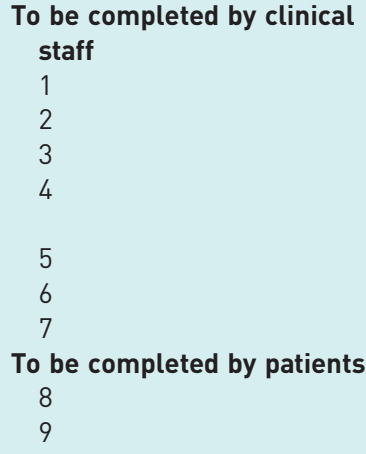

\author{
Patient information, diagnostic evaluation and baseline medical history \\ Physical examination of lungs and heart \\ Physical examination of upper airways and ears \\ Growth measurements and clinical tests (lung function, imaging, \\ microbiology) \\ Hospitalisations and treatment (surgeries, medication, physiotherapy) \\ Clinical symptoms (version A) \\ Environment and lifestyle (version A) \\ Clinical symptoms (version B) \\ Environment and lifestyle (version B)
}


TABLE 2 Content categories of the standardised pulmonary ciliary dyskinesia (PCD) follow-up form (FOLLOW-PCD version 1.0) and degree of agreement of the working group members for their inclusion

\section{Module and content category Main included components}

Degree of group agreement ${ }^{\#} \%$

\section{Module 1}

Demographic information

Diagnostic status

Diagnostic tests performed and results

Differential diagnosis

Baseline medical history

\section{Non-PCD-related} comorbidities

Family history

\section{Module 2}

Vital signs

Clinical examination

Lung auscultation

Heart auscultation

\section{Module 3}

Examination of the nose

Examination of the sinuses

Examination of the ears

\section{Module 4}

Growth measurements

Lung function

Imaging (date and findings)

\section{Microbiology}

\section{Module 5}

Hospitalisations and surgeries
Date of birth, sex, ethnicity

98-100

93-95

Current diagnosis and date, other ciliopathies

nNO measurement: date, result, information on equipment and manoeuvre

95-100

EM: date, sample quality, EM phenotype and \% of affected cilia, ciliary culture

HSVM: date, sample quality, beat frequency, beat pattern, ciliary culture

Genetic analysis: date, technique, genes tested, result, variant nomenclature used

Immunofluorescence: date, absent proteins, positive proteins

Immunodeficiency (dates and results of test performed)

$83-100$

Cystic fibrosis (dates and results of test performed)

Detailed history of neonatal period (symptoms, management, gestational age, birth weight, information on breastfeeding)

Laterality defects (laterality status and cardiovascular malformations, tests and dates of diagnosis)

Fertility problems (test performed, date and result, number of own children)

Retinitis pigmentosa (date of diagnosis)

Hydrocephalus (test performed, date and result)

Kidney problems (problem description, date of diagnosis)

Gastrointestinal problems (problem description, test performed, date of diagnosis)

Upper and lower respiratory symptoms loverview of selected characteristics from the past history, e.g. age of symptom onset, bronchiectasis location and age of diagnosis, pathogens and duration of chronic upper or lower respiratory colonisation, level of hearing impairment and date of evaluation)

Previous surgical interventions related to PCD

Other PCD-related problems

Information on atopic diseases, e.g. asthma, hay fever, atopic dermatitis, tests performed and results, medication allergies

Consanguinity, PCD in the family, family history of other relevant manifestations

$98-100$

Respiratory rate, $\mathrm{S}_{\mathrm{pO}_{2}}$

e.g. signs of dyspnoea, clubbing

Pathological sounds and location

Description of pathological findings

93-95

95-100

93-95

80-93

Discharge and aspect, mucosa, polyps, other findings

Facial pain/sensitivity

Discharge and aspect, otoscopy, audiometry and tympanometry findings

$80-95$

93-95

95-100

Height, weight, date of measurement

$98-100$

Spirometry (date and several indices)

93-100

Plethysmography (date and several indices)

Lung diffusion testing (date and $D_{\text {Lco }}$ result)

Inert gas washout measurements (date, type of test, type of gas and concertation,

number of trials, several indices)

Exhaled breath analyses (date and $F_{\text {eNo }}$ result)

Information on bronchodilation testing (pre- and post-results)

Information on quality of test performed and references used

Chest radiography (date, findings, e.g. atelectasis, infiltrations, bronchial thickening)

Chest CT (date, findings, e. $g$ bronchiectasis, atelectasis, infiltrations, lobar collapse, mucus plugging, location of bronchiectasis, Brody II score)

Lung MRI (date, findings, e.g bronchiectasis, atelectasis, infiltrations, lobar collapse, mucus plugging, location of bronchiectasis)

Sinus radiography (date and findings)

Sinus CT (date, findings and location: aplasia, hypoplasia, bone thickening,

Lund-Mackay score)

Date of culture, type of sample, isolated pathogens, antibiotic resistance

95-100

93-95

Reason for hospitalisation and numbers of hospitalisation days 


\section{TABLE 2 Continued}

\section{Module and content category}

Medication

Physiotherapy

\section{Module 6}

Symptoms from the upper airways and ears

Symptoms from the lower airway

General symptoms

\section{Module 7}

Daily activities

Smoking

Nutrition

Environment
Degree of group agreement ${ }^{\#} \%$

Antibiotics for acute infection (diagnosis, antibiotic class, route, length of treatment)

93-100

Prophylactic antibiotics (antibiotic class, route, dosage, start date)

Oxygen supplementation/ventilation (type and frequency of ventilation)

Other prescribed medication (e.g. bronchodilators, corticosteroids)

Relevant vaccinations (type and date of flu and pneumococcal vaccine)

Upper airways management (type and frequency of intervention)

Lower airways management (type and frequency of interventions performed routinely and during exacerbations, nebulisation of lower airways, routine visits to professional physiotherapist)

Compliance to treatment

Exercise recommendations

Description, severity and periodicity:

chronic nose symptoms

headaches

ear symptoms

snoring

Description, severity and periodicity:

cough

sputum production

wheezing

shortness of breath

chest pain

e.g. fever

Information on working status, days off work/school because of PCD, sports

Active, passive, type and frequency

93-95

Appetite, dietary supplements

Information on neighbourhood traffic, condensation/mould in the house

$85-93$

$80-85$

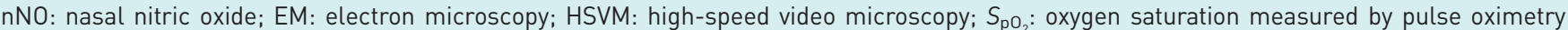
$D_{\mathrm{LCO}}$ : diffusing capacity of the lung for carbon monoxide; $F_{\mathrm{eNO}}$ : exhaled nitric oxide fraction; $\mathrm{CT}$ : computed tomography; $\mathrm{MRI}$ : magnetic resonance imaging. " : range of degree of agreement for the different components of the content category.

pseudo-anonymised data and keep personal information (e.g. names, addresses) separately, held locally at each centre. Data sharing agreements with centres who use the online REDCap platform leave all rights with data contributors (supplementary material).

Data collected on the REDCap platform can easily be extracted and imported into national registries or used for local analyses and clinical audits. Centres contributing to the European Reference Network-Lung PCD registry can easily transfer relevant variables from FOLLOW-PCD to the registry in order to avoid duplication of data entry efforts [8]. For centres contributing to the international PCD (iPCD) cohort, data are automatically linked between iPCD and FOLLOW-PCD, allowing the combination of retrospective and prospective data for certain analyses [21-24].The iPCD cohort aims to use FOLLOW-PCD for all patients who are in clinical follow-up.

\section{Discussion}

This article describes the development and content of a standardised form for collection of clinical data during routine care of PCD patients. The comprehensive clinical form allows collection of standardised routine care data to use in clinical decision-making and in local and collaborative research in observational studies and clinical trials. FOLLOW-PCD has a modular structure to allow flexible use based on the local practice of each centre. It also contains patient questionnaires focusing on symptoms and lifestyle.

The large and interdisciplinary working group and the transparent and inclusive development process are major strengths of this project. Experts in the field of PCD diagnosis, management and research joined forces to develop an instrument, which can be used in clinical practice and for research. We took into consideration feedback of patient representatives as well as needs of different clinical settings, e.g. paediatric and adult, larger and smaller centres. The forms allow the collection of patient-reported data on 


\section{BOX 2 FOLLOW-PCD: how to contribute}

How to participate in the pilot phase

Centres that wish to participate to the project and pilot the form should contact the study team (pcdaispm.unibe.ch). We will provide advice where additional ethical approval is needed. Centres will be asked to sign a data agreement that leaves them all rights to their data. The centres will then receive a password to access the online software REDCap and they will be able to enter their data directly. We will provide technical support for the whole process

How to access data

Centres using the electronic form on REDCap will have constant access to their datasets and can export them directly in various formats for local analyses or transfer to national registries. We have developed detailed instructions to simplify the data extraction procedure

\section{How to translate the questionnaire}

Centres that wish to use the patient questionnaire in another language should contact the study team who will assist in organising the standardised translation process

Researchers who want to use the collected data can propose a topic and a concept sheet describing the planned analyses and publication. Concept sheets have to be approved by centres who want to contribute data to the proposed analysis. Centres who want to participate sign a publication agreement. The study team will then prepare a dataset for the proposed analysis and will work closely with the lead researchers, offering methodological input and support

For further details, contact pcddaispm.unibe.ch

symptoms and on lifestyle. This type of data has not been collected before, and could contribute to understand long-term prognosis and some of the mechanisms behind the clinical variability of PCD. The form is extensive, but has a modular structure allowing centres to choose which modules they want to use and by whom they should be filled in (e.g. physicians, physiotherapists, patients). Implementation in clinical practice might be a lengthy process, particularly the integration of the form into electronic patient record system. In the meantime, the REDCap database allows electronic data entry in real-time.

Data collected using FOLLOW-PCD is available in real-time for national and international monitoring and research. Collaborating centres can easily extract their data and analyse them locally to observe trends (e.g. on bacterial resistance) or to audit their practice (e.g. on prescribed medication or physiotherapy). Clinical teams can then adjust local practice based on their results. It will also allow comparison of care practice between centres at a national or European level for clinical benchmarking of centres. Standardised data can be used for local and collaborative research. The level of detail depends on how extensively each centre uses the form, but ideally, there will be no need for researchers to go back to patient charts to retrospectively retrieve data. In addition, data include patient-reported information on symptoms and lifestyle, which have not been collected in a standardised way in the past. Future analyses can compare different PCD phenotypes, age-related variability and the factors that influence prognosis. In addition, standardised routine observational data will improve the identification of eligible patients for collaborative clinical trials.

The value of standardised data collection for routine clinical data is not a new concept. For many years, clinicians have made efforts to improve their local patient forms and electronic systems to improve care of patients with chronic diseases [25]. For rare diseases, this is particularly important [26, 27]. CF is a characteristic example of a rare disease that has benefited greatly from large long-standing registries [28-30]. However, registries usually collect a minimal dataset and need to be completed in addition to regular clinical forms increasing the workload of healthcare professional. FOLLOW-PCD allows to record all data directly during routine clinical assessments in a standardised way. It is not yet another international registry, but an instrument for the documentation of patient visits in hospital records, and ideally will soon be integrated into the clinic information systems and replace the currently available data entry fields, which vary significantly between centres. Its widespread use in the future will be crucial for improving research and care for patients with PCD.

\section{Next steps}

The original versions of the follow-up form and questionnaires are in English. The patient questionnaires are currently being translated into German and French. Translations into Norwegian, Danish, Dutch, Turkish, Greek, Spanish and Arabic are planned. For each language, two native speakers with proficiency in English and experience in PCD or patient-reported symptoms translate the questionnaire independently. A back-translation ensures that each language version remains true to the original. The translation process is organised by the managing team at ISPM Bern. The questionnaires will be validated in prospective collaborative studies. 
The clinic forms will be piloted in several paediatric and adult PCD outpatient clinics, to test the instruments in real-life conditions and identify items that need to be added or eliminated or if variables must be recoded or further clarified. We plan to pilot the form for a period of 6-12 months in interested centres including different countries and centres of various sizes to make sure it fits different settings (box 2). Our aim is to enrol $\sim 100-200$ patients from five or more countries. We will invite a broad spectrum of centres to make sure we can pilot all aspects of the instrument with sufficient numbers of patients. This includes paediatric and adult centres, specialised pulmonology and ear, nose and throat clinics and centres where certain forms are completed by specialised team members (e.g. physiotherapists) or by patients instead of physicians. We will analyse the data collected during the pilot phase together to evaluate rate of completion of each variable. We will collect comments by the clinical teams of participating centres using a pre-designed form and by participating patients, through the patient questionnaire and the PCD patient organisations. The comments will aim to identify missing items and items that need clarification or recoding. In addition, we will ask if there are redundant items that are not clinically useful or take too much time to complete. Based on the results from the piloting phase, we will suggest changes to the form and use further rounds of the Delphi process, to develop a refined form (FOLLOW-PCD version 2.0).

\section{Conclusion}

In a large, international and multidisciplinary group of PCD experts, we developed FOLLOW-PCD, a standardised clinical PCD form and patient questionnaire. The form will be adapted after piloting in different settings and we encourage and facilitate the translation of the questionnaires into multiple languages. FOLLOW-PCD will facilitate quality research based on prospective standardised data from routine care, which can be pooled between centres, to provide first-line and real-time evidence for clinical decision-making.

Acknowledgements: We want to thank the extended BEAT-PCD working group for their participation in the development of the form and Christopher Ritter (Institute of Social and Preventive Medicine, University of Bern, Switzerland) for his editorial suggestions to this manuscript.

The BEAT-PCD FOLLOW-PCD working group: I. Amirav, Canada; J. Barben, Switzerland; L. Baynton, UK; M. Boon, Belgium; C. Casaulta, Switzerland; S. Castillo, Spain; A. Clement, France; E. Dehlink, Austria; E. Eber, Austria; A. Escribano, Spain; E. Escudier, France; H. Georgy, Israel; M. Goutaki, Switzerland; F. Halbeisen, Switzerland; A. Harris, UK; C. Hogg, UK; I. Honore, France; C. Jackson, UK; A. Jung, Switzerland; B. Karadag, Turkey; R. Koerner-Rettberg, Germany; C.E. Kuehni, Switzerland; R. Lazor, Switzerland; M. Legendre, France; J.S. Lucas, UK G. Marsh, UK; B. Maitre, France; E. Moya, UK; A. Moreno, Spain; K.G. Nielsen, Denmark; J-F. Papon, France; M. Price, Germany; T. Romero, Spain; B. Rubbo, UK; N. Rumman, Palestine; N. Schwerk, Germany; L. Schofield, UK; A. Shoemark, UK; G. Thouvenin, France; H. Wilkins, UK; Z. Zivkovic, Serbia.

Author contributions: M. Goutaki and C.E. Keuhni developed the concept and designed the study. M. Goutaki led the multidisciplinary expert group, and drafted and revised the standardised follow-up form. All authors contributed significantly to the development of the form, revised the manuscript and approved the final version. M. Goutaki and C.E. Kuehni wrote the manuscript and take final responsibility for the contents.

Conflict of interest: M. Goutaki has nothing to disclose. J-F. Papon has nothing to disclose. M. Boon reports COST BEAT-PCD Action (BM 1407) during the conduct of the study, and Postdoc KOOR funding from the University Hospital Leuven and MyCyFAPP Horizon 2020 EU funding outside the submitted work. C. Casaulta has nothing to disclose. E. Eber has nothing to disclose. E. Escudier has nothing to disclose. F.S. Halbeisen has nothing to disclose A. Harris has nothing to disclose. C. Hogg has nothing to disclose. I. Honore has nothing to disclose. A. Jung has nothing to disclose. B. Karadag has nothing to disclose. C. Koerner-Rettberg has nothing to disclose. M. Legendre has nothing to disclose. B. Maitre has nothing to disclose. K.G. Nielsen has nothing to disclose. B. Rubbo has nothing to disclose. N. Rumman has nothing to disclose. L. Schofield has nothing to disclose. A. Shoemark has nothing to disclose. G. Thouvenin has nothing to disclose. H. Wilkins has nothing to disclose. J.S. Lucas reports COST Action BM1407 BEAT-PCD during the conduct of the study. C.E. Kuehni has nothing to disclose.

Support statement: This study was supported by the Swiss National Foundation (SNF 320030_173044) and the Bern Lung League. The researchers participate in the network COST Action BEAT-PCD: Better Evidence to Advance Therapeutic options for PCD (BM 1407). J-F. Papon, E. Escudier, M. Legendre, B. Maitre and I. Honore participate in the RaDiCo project funded by the French National Research Agency under the programme "Investments for the Future" (cohort grant agreement ANR-10-COHO-0003). Funding information for this article has been deposited with the Crossref Funder Registry.

\section{References}

1 Lucas JS, Walker WT, Kuehni CE, et al. Primary ciliary dyskinesia. In: Cordier J-F, ed. Orphan Lung Diseases (ERS Monograph). Sheffield, European Respiratory Society, 2011; pp. 201-217.

2 Strippoli MP, Frischer T, Barbato A, et al. Management of primary ciliary dyskinesia in European children: recommendations and clinical practice. Eur Respir J 2012; 39: 1482-1491.

3 Kuehni CE, Frischer T, Strippoli MP, et al. Factors influencing age at diagnosis of primary ciliary dyskinesia in European children. Eur Respir J 2010; 36: 1248-1258.

4 Lucas JS, Chetcuti P, Copeland F, et al. Overcoming challenges in the management of primary ciliary dyskinesia: the UK model. Paediatr Respir Rev 2014; 15: 142-145. 
5 Goutaki M, Eich MO, Halbeisen FS, et al. The Swiss Primary Ciliary Dyskinesia registry: objectives, methods and first results. Swiss Med Wkly 2019; 149: w20004.

6 Barbato A, Frischer T, Kuehni CE, et al. Primary ciliary dyskinesia: a consensus statement on diagnostic and treatment approaches in children. Eur Respir J 2009; 34: 1264-1276.

7 Kuehni CE, Goutaki M, Rubbo B, et al. Management of primary ciliary dyskinesia: current practice and future perspectives. In: Chalmers JD, Polverino E, Aliberti S, eds. Bronchiectasis (ERS Monograph). Sheffield, European Respiratory Society, 2018; pp. 282-299.

8 Werner C, Lablans M, Ataian M, et al. An international registry for primary ciliary dyskinesia. Eur Respir J 2016; 47: 849-859.

9 Halbeisen FS, Jose A, de Jong C, et al. Spirometric indices in primary ciliary dyskinesia: systematic review and meta-analysis. ERJ Open Res 2019; 5: 00231-2018.

10 Goutaki M, Meier AB, Halbeisen FS, et al. Clinical manifestations in primary ciliary dyskinesia: systematic review and meta-analysis. Eur Respir J 2016; 48: 1081-1095.

11 Feder SL. Data quality in electronic health records research: quality domains and assessment methods. West J Nurs Res 2018; 40: 753-766.

12 Knake LA, Ahuja M, McDonald EL, et al. Quality of EHR data extractions for studies of preterm birth in a tertiary care center: guidelines for obtaining reliable data. BMC Pediatr 2016; 16: 59.

13 Rubbo B, Behan L, Dehlink E, et al. Proceedings of the COST action BM1407 inaugural conference BEAT-PCD translational research in primary ciliary dyskinesia - bench, bedside, and population perspectives. BMC Proc 2016; 10: 66 .

14 Farley H, Rubbo B, Bukowy-Bieryllo Z, et al. Proceedings of the 3rd BEAT-PCD Conference and 4th PCD Training School. BMC Proc 2018; 12: 64.

15 Halbeisen F, Hogg C, Alanin MC, et al. Proceedings of the 2nd BEAT-PCD conference and 3rd PCD training school: part 1. BMC Proc 2018; 12: 1.

16 Dell SD, Leigh MW, Lucas JS, et al. Primary ciliary dyskinesia: first health-related quality-of-life measures for pediatric patients. Ann Am Thorac Soc 2016; 13: 1726-1735.

17 Lucas JS, Behan L, Dunn Galvin A, et al. A quality-of-life measure for adults with primary ciliary dyskinesia: QOL-PCD. Eur Respir J 2015; 46: 375-383.

18 Behan L, Leigh MW, Dell SD, et al. Validation of a health-related quality of life instrument for primary ciliary dyskinesia (QOL-PCD). Thorax 2017; 72: 832-839.

19 Behan L, Leigh MW, Dell SD, et al. Validation of paediatric health-related quality of life instruments for primary ciliary dyskinesia (QOL-PCD). Pediatr Pulmonol 2018; 54: 2011-2020.

20 Harris PA, Taylor R, Thielke R, et al. Research electronic data capture (REDCap) - a metadata-driven methodology and workflow process for providing translational research informatics support. J Biomed Inform 2009; 42: 377-381.

21 Goutaki M, Maurer E, Halbeisen FS, et al. The international primary ciliary dyskinesia cohort (iPCD Cohort): methods and first results. Eur Respir J 2017; 49: 1601181.

22 Goutaki M, Halbeisen FS, Spycher BD, et al. Growth and nutritional status, and their association with lung function: a study from the international Primary Ciliary Dyskinesia Cohort. Eur Respir J 2017; 50: 1701659.

23 Halbeisen F, Goutaki M, Maurer E, et al. Evolution of primary ciliary dyskinesia (PCD) diagnostic testing in Europe. Eur Respir J 2017; 50: PA1846.

24 Halbeisen FS, Goutaki M, Spycher BD, et al. Lung function in patients with primary ciliary dyskinesia: an iPCD Cohort study. Eur Respir J 2018; 52: 1801040.

25 Jones P, Harding G, Wiklund I, et al. Improving the process and outcome of care in COPD: development of a standardised assessment tool. Prim Care Respir J 2009; 18: 208-215.

26 Kodra Y, Weinbach J, Posada-de-la-Paz M, et al. Recommendations for improving the quality of rare disease registries. Int J Environ Res Public Health 2018; 15: E1644.

27 Lacaze P, Millis N, Fookes M, et al. Rare disease registries: a call to action. Intern Med J 2017; 47: 1075-1079.

28 Mak DY, Sykes J, Stephenson AL, et al. The benefits of newborn screening for cystic fibrosis: the Canadian experience. J Cyst Fibros 2016; 15: 302-308.

29 Taylor-Robinson D, Archangelidi O, Carr SB, et al. Data resource profile: the UK Cystic Fibrosis Registry. Int J Epidemiol 2018; 47: 9-10e.

30 Jackson $\mathrm{AD}$, Goss $\mathrm{CH}$. Epidemiology of $\mathrm{CF}$ : how registries can be used to advance our understanding of the $\mathrm{CF}$ population. J Cyst Fibros 2018; 17: 297-305. 